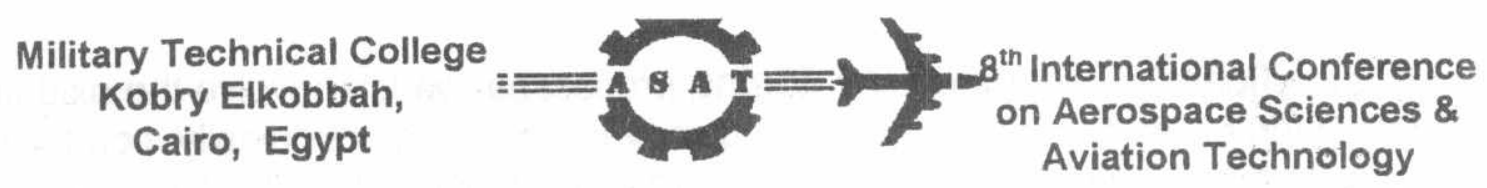

\title{
SYNTHESIS AND CHARACTERIZATION OF ORGANIC POLYMER CONDUCTOR BASED ON POLYANILINE
}

\author{
M.SH.FAYED *, SH.R.GOUDA **, M.A.RADWAN ** AND \\ M.A.BORHAM **
}

\section{ABSTRACT}

Specialty polymers have important commercial markets in the aerospace, information technology, electronic industries and biomedical applications. A number of electoractive polymers have been the object of increasing academic and industrial interest during the past twenty years. Substantial progress has been achieved in the development and characterization of what is called the synthetic metals (or organic polymer conductors). In the frame work, polyaniline was selected in the presented paper, to be synthesized and studied. It was prepared via the catalytic oxidation of aniline in acidic medium using ammonium persulfate in hydrochloric acid. The variation of the electric conductivity of the prepared polymer was correlated with the molar ratio of aniline to to the oxidant under the experimental conditions. 'The polymer was investigated by several physical and analytical techniques, the results of the work were given, explained and discussed.

\section{KEY WORDS}

Polyaniline, organic metals, electric conductivity, chemical oxidation and aromatic amines.

\section{INTRODUCTION}

The application of Specialty polymers in electronics and photonics is extensive, both in a "passive" role -when they act as insulators, adhesives materials for integrated circuit fabrication and encapsulating agents -and in an active way as electronic and photonic conductors or as "active" material in non-linear optics [1]. A number of polymers are electrically conductive or can be made to be conductive by doping with electron donor or acceptor. Applications include: polymeric electrodes for light weight batteries, variable-transmission windows, electro chromic displays, sensors, non-linearoptical materials, electromagnetic shielding, optical fibers and optical information storage[2].Electroactive polymers are usually classified in two large groups according to the mode of their electric transport. One group includes polymers having transport almost exclusively of the ionic type(called polymer elctrolytes or polymer ionics). The other group includes polymeric materials where the transport .mechanism is mainly electronic termed "conducting polymers "

*... Egyptian Military Forces. 
may be significantly modified by doping processes which involve the oxidation or the reduction of the polymer chain. The discovery of the electronically conducting polymers dates few years back when it was announced that the conductivity of polyacetylene could be raised several orders of magnitude by redox processes. Appropriate functionaliztion, doping and its level, controlled synthesis and other factors may make the conducting polymers to find unique applications in advanced technology. It was reported that polyaniline is a peculiar conducting polymer because it is the only one, along with its dervatives, whose electrical properties not only depend on its oxidation state but also on the acidity of the electrolyte medium. Polyariline has been subjected to several electrochemical studies $[3,4]$. In this work, polyaniline was selected to be chemically prepared via its oxidation using ammonium persulphate in an acid medium. The prepared polymer was analyzed and invstigated.

\section{EXPERIMENTAL}

\section{Chemicals:}

Aniline(BDH), ammonium persulphate(Aldrich), hydrochloric acid(EI-Naser), acetone (Prolabo), formamide (Aldrich), dimethylformamide(Aldrich), benzene(adwic), ethanol(prolabo), and dimethylsulfoxide (colinbrook) were used without futher purification except the redistillation of aniline.

\section{Chemical polymerization:}

The chemical polymerization of distilled aniline was performed by its oxidation with ammonium persulphate in the presence of hydrochloric acid as follows:

Ammonium persulphate (27.5g.) was dissolved in hydrochloric acid $(350 \mathrm{ml}, 1 \mathrm{M})$ and was cooled to $0^{\circ} \mathrm{c}$ using an ice bath. Redistilled aniline $(11.7 \mathrm{ml})$ was dissolved in hydrochloric acid $(700 \mathrm{ml}, 1 \mathrm{M})$ and was also cooled to $0^{\circ} \mathrm{c}$. Few drops of ferrous sulphate aqueous solution (2\%) were added to the cold aniline solution. The ammonium persulphate solution was gradually added to the aniline solution all over a period of 15 minutes while cooling. After one hour of vigorous stirring of the cold mixture, it was transferred to a freezer for about two hours, then an excess ammount of aniline $(6 \mathrm{ml})$ was added. A green precipitate was formed, filtered, washed and dried in an oven at $50^{\circ} \mathrm{c}$ for three days.

\section{Instrumentation:}

Thermalgravimetric analysis (TGA) fig1 of the product was carried out with a rate of $15^{\circ} \mathrm{c}$ per minute, the test was conducted in the temperature range of $0-450^{\circ} \mathrm{C}$ using NETZSCH TG 209 instrument, (West.Germany). Infrared absorption spectrum was carried out by dispersing the samples in $\mathrm{KBr}$ and measured on a Perkin-Elmer model 3087 spectrophotometer, in wave number range of $500-4000 \mathrm{~cm}^{-1}$. The visible (UV)absorption spectrum of the polymer solutions were determined using shimmadzu UV-visible-NIR-160 spectrophotometer (Japan). The electric conductivity was 
determined using an electronic resistivity apparatus model 120 Hwellet Packard (France). The test was carried out on a glass substrate with squared shape of $24 \mathrm{~mm} . \times 24 \mathrm{~mm}$ dimensions.

\section{RESULTS AND DISCUSSIONS}

Polyaniline can be chemically synthesized by aniline polymerization using various oxidizing agents.Genies et.al [3].compared some oxidizing agents such as permangate, dichromate, iodate and persulphate for the polymerization of aniline in $\mathrm{NH}_{4} \mathrm{~F} .2 .3 \mathrm{HF}$ and found that a high yield of polyaniline was obtained using the persulphate oxidizing agent. So in this work, ammonium persulphate, was selected to be used in the synthesis under discussion. In spite of its study by severall workers, polyaniline, still, has an attracting characterstics due to its complicated electronic structure. It can be considered as the oldest electoactive synthetic polymer but up till now recent publications are disscussing its synthesis, structure and applications.In an early work [5]., it was reported that polyaniline was an octamer whose color depend on the nature of quinione imine (Q) moities in the backbone. Thus " lucoemeraldine " had no $Q$ " protoemeraldine " had one Q, "emeraldine " had two Q " "nigranline" had three $Q^{.5}$, and "pernigraniline" had four $Q^{.5}$, according to the oxidation state. On the other hand, within the last fifteen years, Mac Diarmid et al [6]. classified the structure of polyaniline as a completely reduced form "1", a completely oxdized form " 2 ", imine and amine " $a$ " and a protonated form " $s$ " as follows:

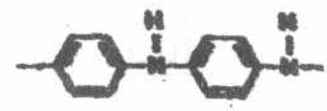

(1A)

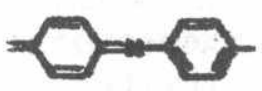

(2A)

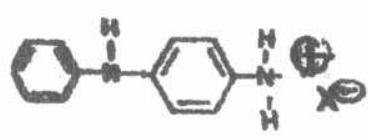

(1S)

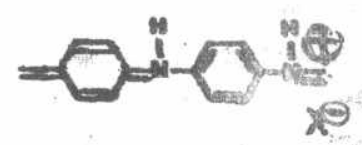

(25)

So polyaniline is a peculiar polymer because its polymerization mechanism, structure, and applications are still in field. In this paper a brief account of primary results on the preparation of the polymer and an investigation of its physical, spectroscopic and electrical properties as functions of molar ratio of oxidant to aniline and doping level was carried out. The possible mechanism of aniline oxidation is the loss of an electron from the lone pair to form the corresponding radical cation which can be represented by the equation:

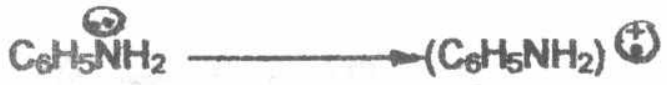


The formed radical cation can attack another aniline molecule at the ortho- and frara positions to form PANI in its final stage. The para attack is believed to be the most dominant from the steric factor point of view. The extent of oxidation depending on the ratio of used oxidizing agent and time can lead to a variable content of the coxiclized form containing the quinone moity in the polymer backbone. In table1, the electrical conductivity and the polymer weight were dependant on the reactants molar ratio (aniline:oxidizing agent), this electric conductivity of the samples was calculated according to the following

$$
\sigma=R \times d \times C / L
$$

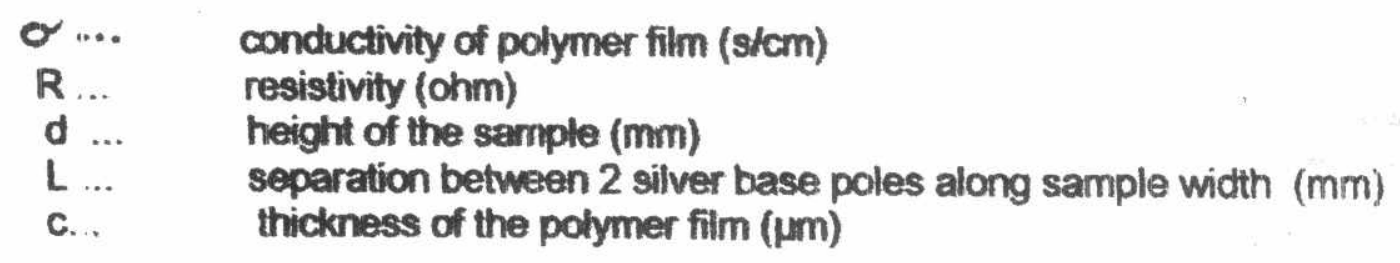

Table1. Dependence of the weight of the product and the conductivity of the prepared Polyaniline on the molar ratio of the reactants

\begin{tabular}{|c|c|c|c|}
\hline Sample & $\begin{array}{c}\text { Molar ratio } \\
\text { Aniline/oxidant }\end{array}$ & $\begin{array}{c}\text { Conductivity } \\
\text { (S/cm) }\end{array}$ & $\begin{array}{c}\text { Polymer weight } \\
(\mathrm{g})\end{array}$ \\
\hline S1 & $1: 1$ & 1.1 & 7.9 \\
\hline S2 & $1: 2$ & 1.5 & 8.2 \\
\hline S3 & $1: 3$ & 3.8 & 9.1 \\
\hline S4 & $1: 4$ & 29.7 & 9.9 \\
\hline S5 & $1: 5$ & 34.4 & 10.2 \\
\hline S6 & $1: 6$ & 60 & 11.4 \\
\hline S7 & $1: 7$ & 61.1 & 11.7 \\
\hline
\end{tabular}

From table1 and the graphical repesentation fig4, it is clear that the conductivity increases as the molar ratio increases. The samples 6 and 7 ( $\$ 6$ and $\$ 7$ ) preprared with molar ratio of aniline:oxidant $1: 6-7$ have shown higher conductivities compared with others. Sample S6 was taken in the following characterisation of the conclucting polymer because its conductivity value $60 \mathrm{scm}^{-1}$ located in the border region between semiconductor and metallic conductors [7]. This may be attributed to the maximum number of quinone ring that can be produced under these conditions. But that is not sufficient for this higher conductivity since, it is reported [8]. that the totally oxidized polyaniline is an insulator. So the role of $\mathrm{HCl}$ is the protonation of polyaniline to form the corresponding salt. This takes place preferentially on the imine nitrogen $(-N=)$ atoms as follows:

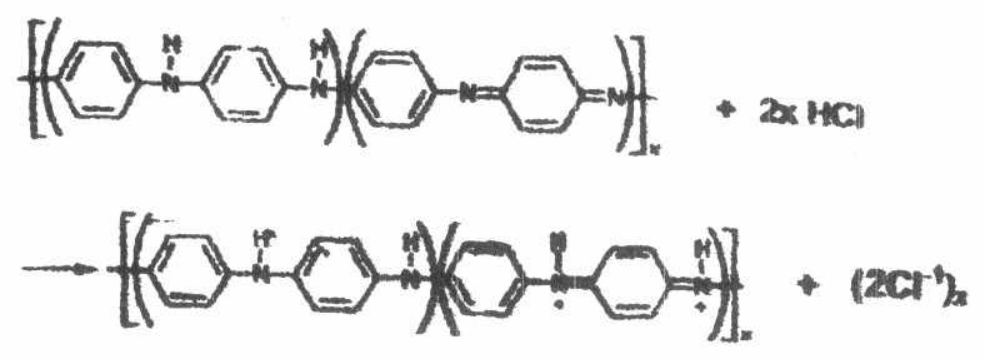


It is the protonation reaction that induce a large increase in the polymer conductivity observed by the p-doping (oxidation) process. This p-doping leads to the formation of a positive charge localised in the polymer chain accompained by the transformation of the aromatic structure towards the quinoid form. The increase in the oxidizing agent leads to a corresponding increase in the quinone or imine form, thus enhancing the protonation process, and higher conductivity due to the delocalised pi-electrons in the structure as the doping level increases. The conductivity may approach that of metals shown in fig5. In such case the conductivity can be looked as a result of both the conjugation and the regularity of the polymer structure. In the same time the p-doping (oxidation) implies the diffusion of the oppositely charged chloride counterions from the medium to the polymer. Threfore by that doping, the polymer experiences, in addition to its electronic structure, an increase in mass as it is shown in table1.

IR- spectrum of sample 6 taken as the one with higher conductivity is shown in fig2. It reveals the existence of the bands in the region of $1400-1240 \mathrm{~cm}^{-1}$ which correspond to the $\mathrm{C}-\mathrm{N}$ stretching characteristic of the aromatic amines. The peak at $1160 \mathrm{~cm}^{-1}$ correspond to $\mathrm{C}=\mathrm{N}$ in the quinone imine moity. The aromaticity of the polymer can be shown by peaks at $745 \mathrm{~cm}^{-1}$ and $695 \mathrm{~cm}-1$. The p-disubstituted benzene structure is concluded from the peak at $830 \mathrm{~cm}^{-1}$.

UV-.absorption peak characteristics of emeraldine base are at $354 \mathrm{~nm}$ and $620 \mathrm{~nm}$ givining rise to deep blue solutions. The formation of emeraldine salt is accompanied by the the disappearance of the peak at $620 \mathrm{~nm}$ due to the rearrangement of the quinoid ring system into a benzoid ring system. the appearance of three new peaks at $320 \mathrm{~nm}, 420 \mathrm{~nm}$ and $830 \mathrm{~nm}$ are associated with this arearrangement. Protonation of the base form results in a green colored emeraldine salt that rapidly precipitates from the acidic solutions. Testing the UV-visible absorbance of the prepared sample S6 fig3 show the existence of the peak of $320 \mathrm{~nm}$, which concides with the above mentioned characteristics.

Thermalgravimetric analysis (TGA) for sample 6 is shown in fig1. The polymer shows one weight loss region at $(40-90)^{\circ} \mathrm{c}$ with $30 \%$ loss in weight, this can be attributed to the removal of residual monomer and water. The second region weight loss located at $267^{\circ} \mathrm{C}\left(250-300^{\circ} \mathrm{c}\right)$ can be explained by the partial decomposition of the organic polymer. The peak located at $224{ }^{\circ} \mathrm{C}$ is the glass transition of this sample with its change from the glass to the rubber like state. This thermal analysis indicates that the sample is stable up to about $265^{\circ} \mathrm{C}$.

The solubility in several solvents was tesred. It was shown that formamide is as good solvent for the prepared sample where as DMF is a poor solvent. Other tested solvents such as ethanol, acetone, benzene can be considered as nonsolvents for the prepared polyaniline at normal temperature

\section{Conclusion:}

Polyanline was prepared by the chemical oxidation of pure aniline using ammonium 
persulfate in an acid medium and ferrous sulfate as a catalyst. The charaterization of the prepared polymer was confirmed by UV-, IR-spectroscopy, thermalgravimetric Solubility and conductivity. The weight of the polymer product and its conductivity varied depending on the molar ratio of aniline to persulfate., The maximum conductivity obtained was $61.1 \mathrm{~S} / \mathrm{cm}$ (at molar ratio $1: 7$ aniline / persulfate ). Polyaniline conductivity $(61.1 \mathrm{~s} / \mathrm{cm})$ makes it very close to metals ( conductivity of metals $10^{2} . \mathrm{s} / \mathrm{cm}$ ) " From the thermagravimetric analysis it is clear that the polymer sample 6 is stable up to about $265^{\circ} \mathrm{c}$. Polyaniline conducting polymer can be applied for coating from solutions due to its solubility at room temperature. . The effect of temperature and and concenteration on the solubility was not studied.The future study will be conccenterated to form a phase diagram and to calculate the polyaniline solubility paramieter in different solvents.

\section{REFERENCES}

1-Cowie,.M.G "Polymers : Chemistry and Physics of Modem Materials" $2{ }^{\text {nd }}$ Ed., Blacki, (1991).

2-Scrosati,B. "Applications of Electroactive Polymers", Chapman and Hall, (1993).

3-Genies. E.M, Boyle.A., Lapkowski.M and Tsintavis.C, "Synthetic metals, 36, 13982, (1980).

4-Kobayashi.T, Toneyame.H and Tamura.H, J Electroanal.Chem.161, 419-23 and 177, 281-91, (1984).

5-Green.A.G, Woodhead.A.E, J.Chem.Soc.,97, 2388, (1910) and 101, 1117, (1912).

6-Mac.Diarmid.A.G., Chiang.J.C, Halpern.M., mol.cryst., 121, 173, (1985).

7-M. Goosey., Speciality polymers, $1^{\text {st }}$ ed.Chapman and Hall, New York, (1987), pag. 85.

8- Bruce, P.G., "Chemistry of solid state Materials: Solid state Electrochemistry" Cambridge univ. press., (1995). 


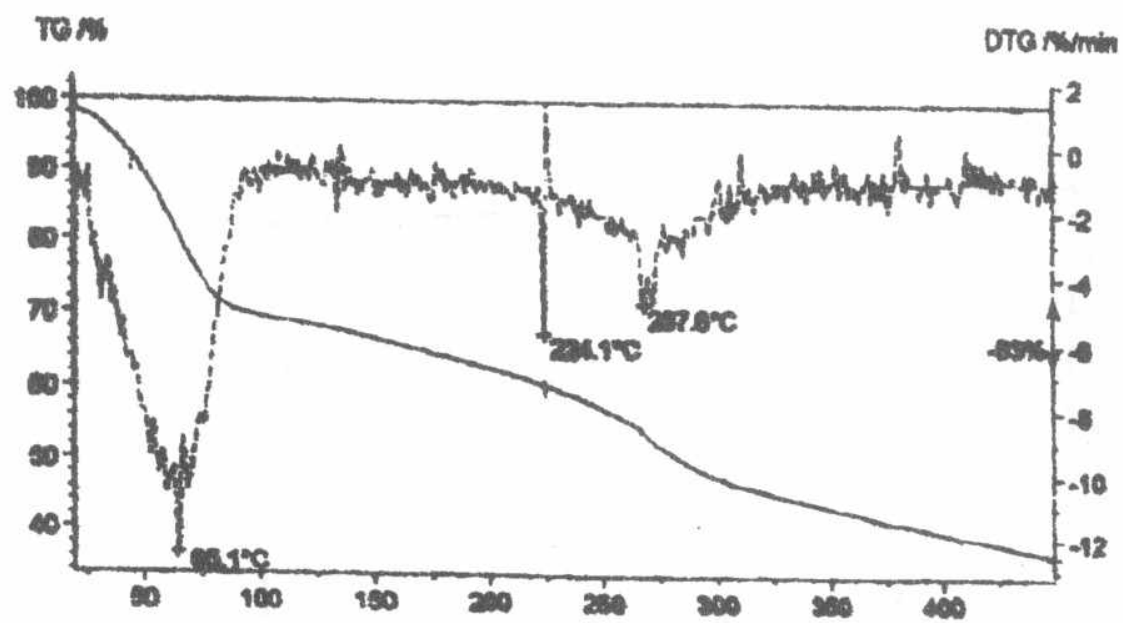

Temperature $/{ }^{0} \mathrm{c}$

Fig1. Thermalgravimetric data of polyaniline of sample 6 one dynamic Segment $15^{\circ} \mathrm{c}$ per min

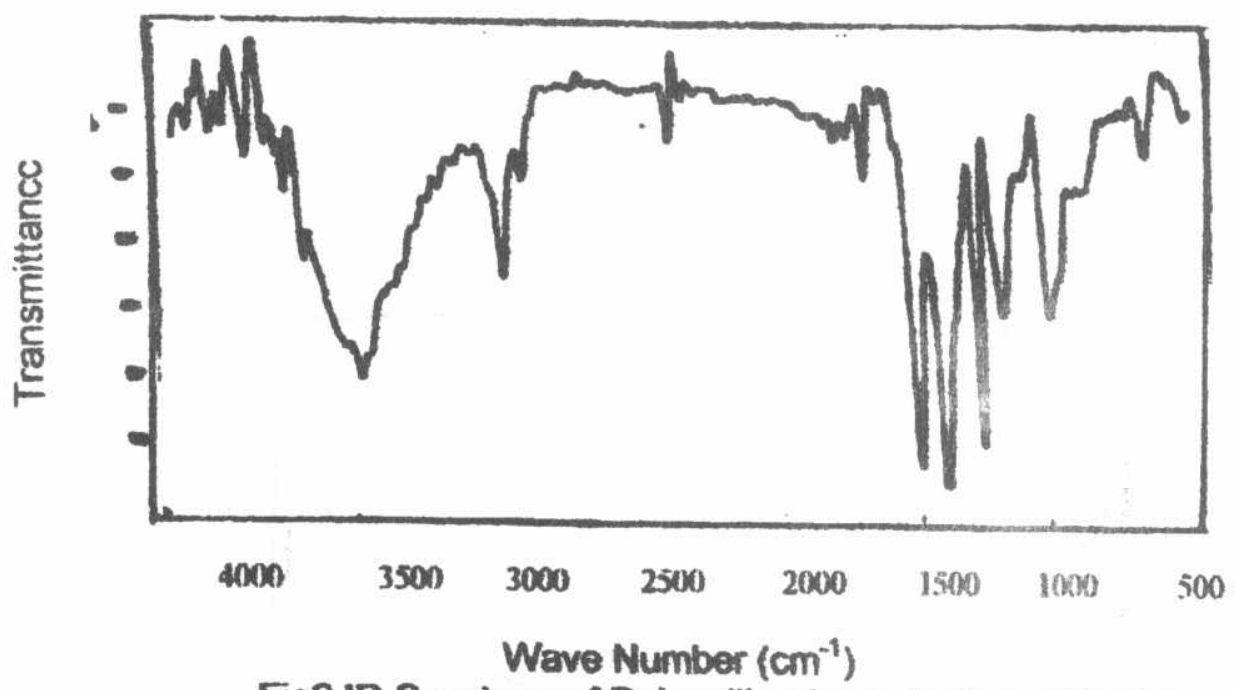

Fig2.IR-Spectrum of Polyaniline (sample 6), in $\mathrm{KBr}$ disk 


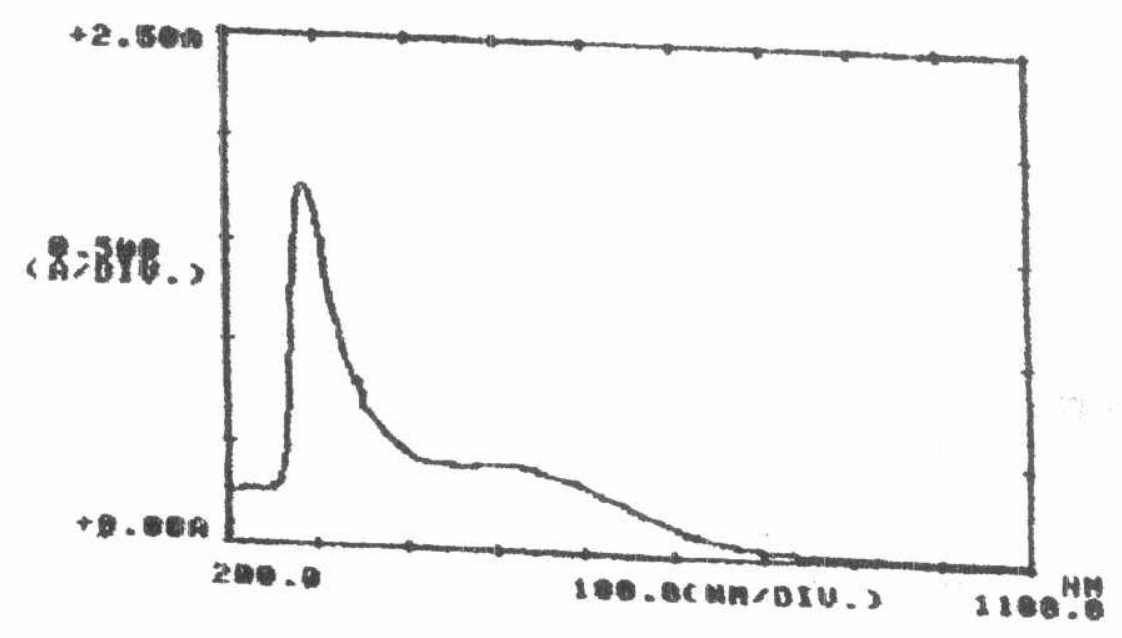

Fig3 UV-visitle absorption of polyaniline (sample 6) diseotred in formanide

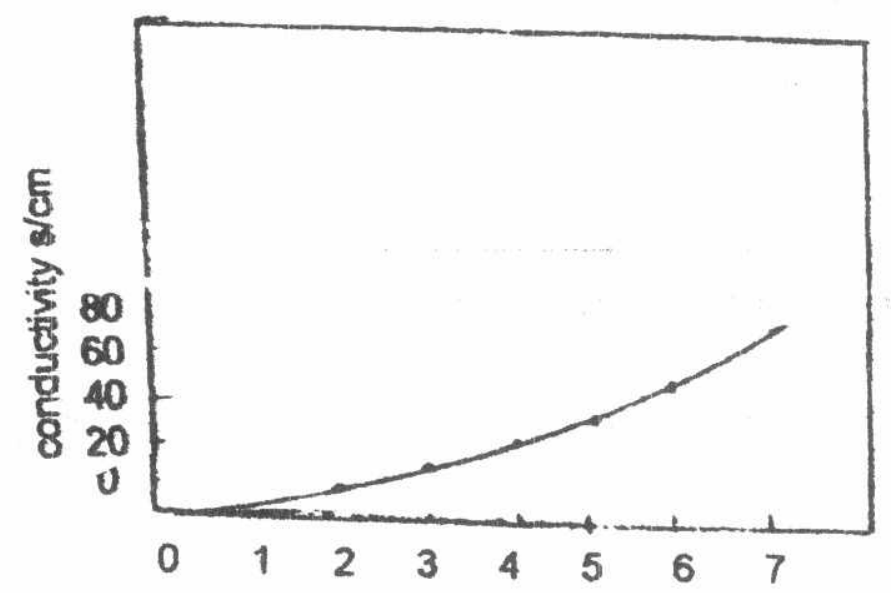

Fig4. The retation between the conductivity of polyanitine samples and the motar ratio of the reactants 


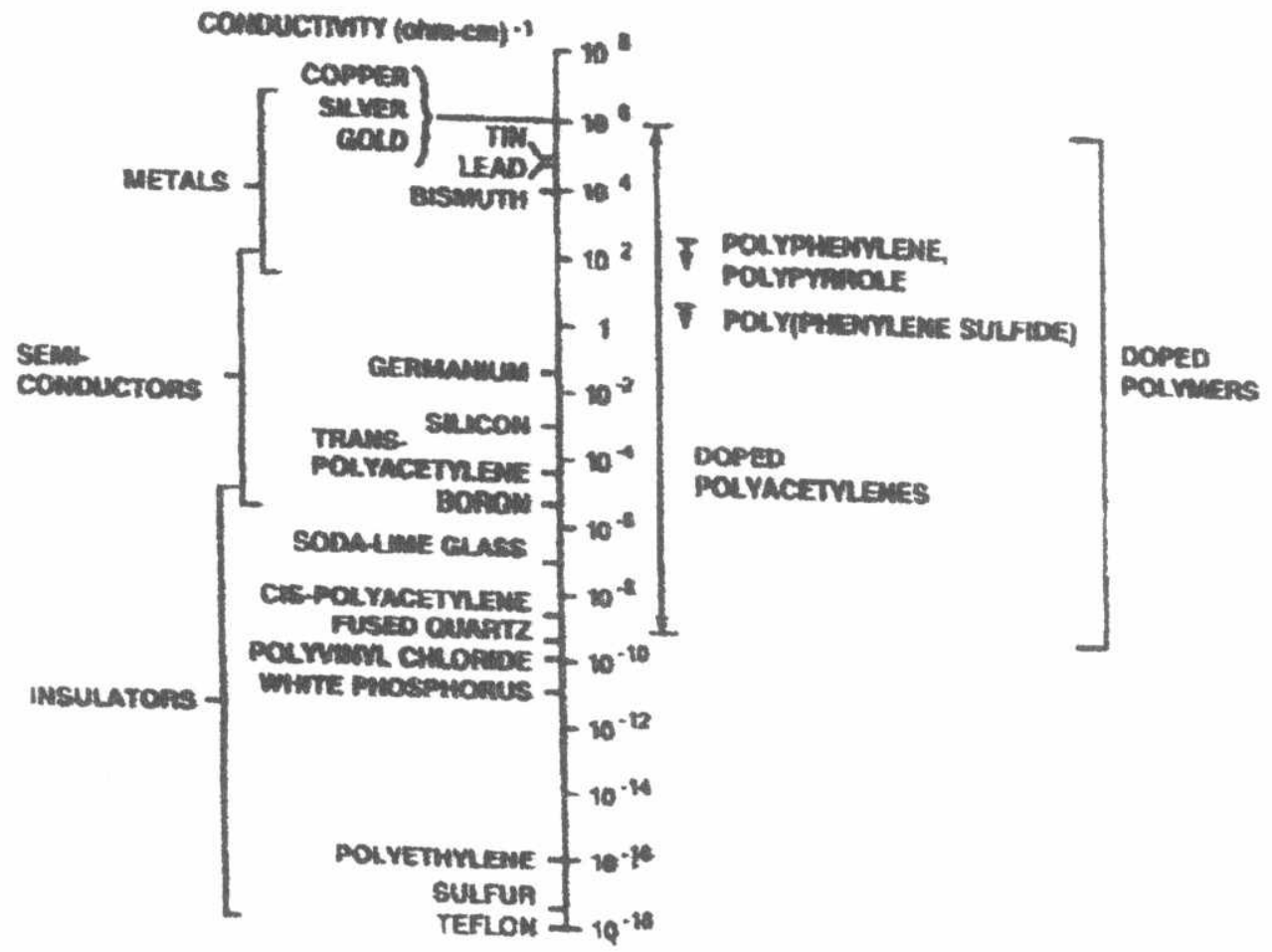

Fig5. The conductivity levels of the different materials 